\title{
Thymoma associated with fatal myocarditis and polymyositis in a 58-year-old man following treatment with carboplatin and paclitaxel: A case report
}

\author{
HIDEFUMI SASAKI, MOTOKI YANO, OSAMU KAWANO, YU HIKOSAKA and YOSHITAKA FUJII \\ Department of Surgery II, Nagoya City University Medical School, Kawasumi 1, \\ Mizuho-cho, Mizuho-ku, Nagoya 467-8601, Japan
}

Received August 31, 2011; Accepted November 8, 2011

DOI: $10.3892 / 01.2011 .501$

\begin{abstract}
We report the case of a 58-year-old male presenting with giant cell myocarditis and myositis associated with thymoma following treatment with carboplatin and paclitaxel. The patient was diagnosed as having stage IVa thymoma. Acetylcholine receptor binding antibody titers were positive at $42 \mathrm{nmol} / \mathrm{l}$, although the patient exhibited no symptoms of myasthenia gravis (MG). The patient was treated with a combination of carboplatin and paclitaxel. However, 18 days following administration of this second cycle of chemotherapy, the patient developed a low-grade fever. Twenty-one days after receiving the second cycle of chemotherapy, the patient was admitted to the Nagoya City University Medical School complaining of general fatigue. Serum levels of creatinine phosphokinase (7,271 U/l), alanine aminotransferase (469 U/1) and aspartate aminotransferase (561 U/l) were elevated. Electromyography revealed no evidence of a neuromuscular junction defect or myopathic process. The patient developed progressive muscle weakness and succumbed to the disease in hospital on day 9. An autopsy revealed thymoma invasion of the left parietal and visceral pleura, pericardium and diaphragm. Numerous skeletal muscle groups and myocardium exhibited diffuse lymphocytic infiltration. Although it has been suggested that myocardial disorders may occur in patients with thymoma and/or MG, the mechanism involved remains unknown. This second report may provide new data regarding giant cell myocarditis and myositis associated with thymoma following treatment with carboplatin and paclitaxel.
\end{abstract}

\section{Introduction}

Polymyositis and myocarditis associated with thymoma are exceptionally rare and are usually accompanied by myasthenia

Correspondence to: Dr Hidefumi Sasaki, Department of Surgery II, Nagoya City University Medical School, Kawasumi 1, Mizuho-cho, Mizuho-ku, Nagoya 467-8601, Japan

E-mail: hisasaki@med.nagoya-cu.ac.jp

Key words: thymoma, myocarditis, myositis, carboplatin, paclitaxel gravis (MG). In 1944, Giordano and Haymondo reported the first autopsied case with the four diseases, MG, thymoma, giant cell polymyositis and cardiomyositis (1). In 1969, Burke and colleagues who had observed ST segment depression and T-wave inversion on the electrocardiogram (ECG) in one case, reported that the patient suddenly died due to rapid progression of heart failure (2). In their study, Namba and colleagues reported 13 cases of giant cell polymyositis, cardiomyositis and thymoma (3). Gidron and colleagues reported a thymoma case with fatal myocarditis following treatment with carboplatin and paclitaxel (4). We experienced a similar case.

\section{Case report}

A 58-year-old male presented to Nagoya City University Medical School, Japan, with stage IVa type B2 thymoma. An asymptomatic, anterior mediastinal mass had been identified 2 years previously upon routine chest X-ray examination, but the patient had not received any treatment prior to presentation. A biopsy was performed at a local hospital and the tumor was diagnosed as a type B2 thymoma. Chest radiography revealed left pleural effusion and a widened mediastinum with multiple chest wall masses. Computed tomography (CT) revealed that the tumor existed in the anterior mediastinum and that multiple dissemination existed in the left thoracic cavity, with pleural effusion (Fig. 1). Acetylcholine receptor binding antibody titers were positive at $42 \mathrm{nmol} / 1$ (normal $<0.3$ nmol/1), although the patient had no symptoms of MG. The study was approved by the Institutional Review Board of the hospital. The patient provided informed consent and received two courses of carboplatin, $550 \mathrm{mg}$ and paclitaxel, $330 \mathrm{mg}$. No problems were experienced following the first cycle of chemotherapy.

However, 18 days after receiving the second cycle of chemotherapy, the patient developed a low-grade fever. Then, 21 days after receiving chemotherapy, the patient was admitted to hospital due to general fatigue. Physical examination was notable for mild diffuse muscle tenderness. Laboratory examination revealed that serum levels of creatinine phosphokinase (7271 U/1; normal, 62-287 U/1), alanine aminotransferase (469 U/1; normal 6-37 U/1) and aspartate aminotransferase (561U/1; normal 10-33 U/1) were elevated. Serum electro- 


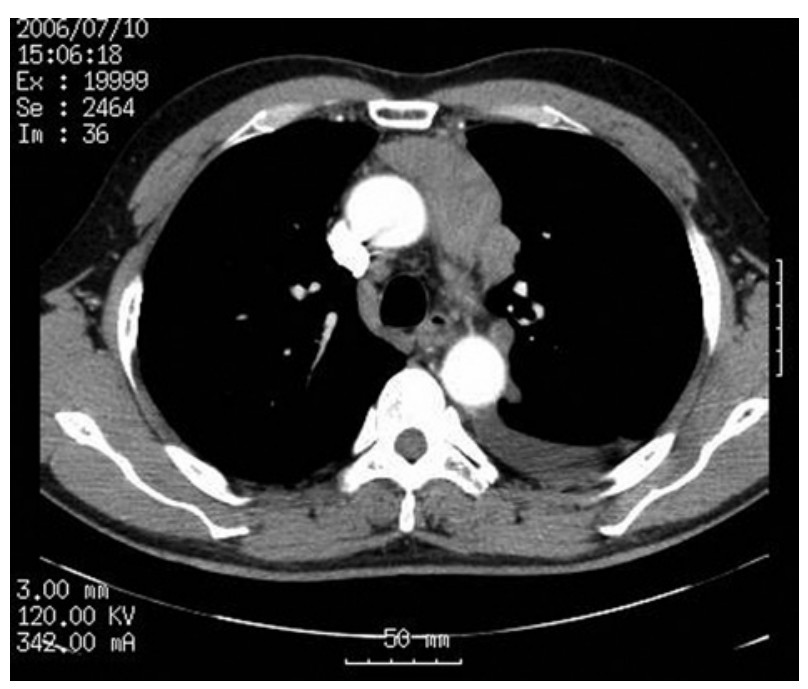

Figure 1. Computed tomography (CT) revealed the anterior mediastinum tumor and multiple dissemination in the left thoracic cavity, with pleural effusion.

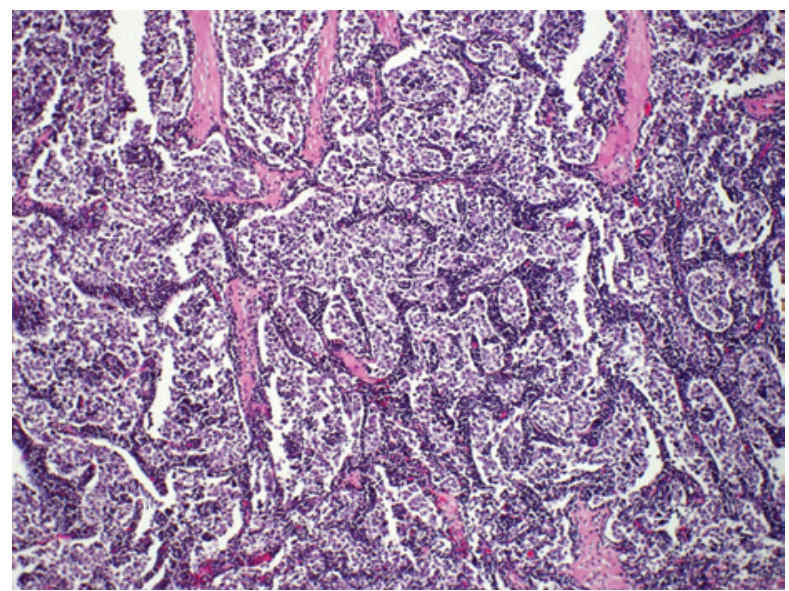

Figure 2. Thymic tumor obtained at autopsy shows type B2 thymoma Histology of thymoma showing epithelial tumor cells with moderate lymphocyte infiltration of macrophages. Multinucleated giant cells also existed in the biceps muscles.

lytes and calcium were normal. Viral titers were negative. An evoked electromyogram showed that electrical activity produced by the skeletal muscles was within the normal range. The patient tested negative for Tensilon (edrophonium chloride). CT revealed that the tumor size had increased. The patient developed progressive muscle weakness and suddenly succumbed in hospital on day 9 .

An autopsy revealed that type B2 thymoma (Fig. 2), invasion of the left pleura, pericardium, diaphragm and multiple dissemination existed in the left thoracic cavity, with pleural effusion. At autopsy the heart weighed $330 \mathrm{~g}$ and there was no evidence of valvular or significant coronary artery disease. Numerous skeletal muscle groups and the myocardium exhibited diffuse lymphocytic infiltration, predominantly by CD8-positive T cells. The inflammatory cells were predominantly mononuclear cells, including lymphocytes, plasma cells and macrophages. Multinucleated giant cells also existed in muscles and myocardium (Fig. 3). In the existing literature,

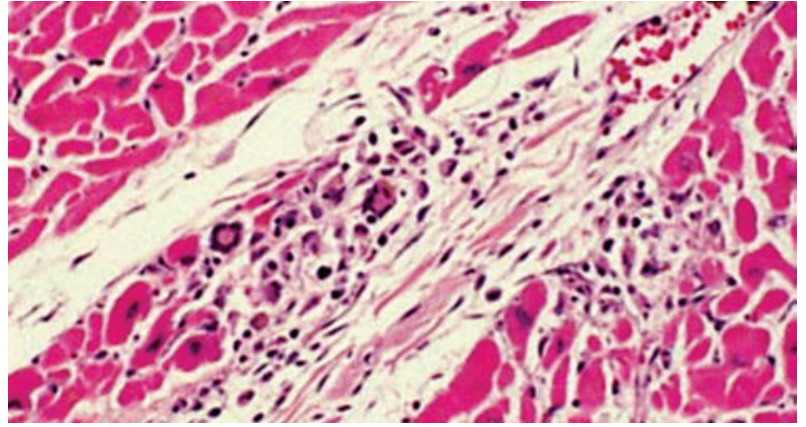

Figure 3. Myocardium obtained at autopsy shows lymphoid cell infiltration and multinucleated giant cells.

certain instances of the syndrome of giant cell myocarditis and polymyositis associated with invasive thymoma have been reported, and this case is also thought to belong to this syndrome.

\section{Discussion}

Thymoma is one of the most common tumors in the anterior mediastinum. Thymoma typically grows slowly when it is at the early stages. Conversely, a number of studies have shown poorer prognosis for patients with invasive thymomas $(5,6)$. Since complete resection is often impossible for advanced-stage thymoma, multimodal treatment strategies are required (7).

Polymyosis and myocarditis associated with thymoma are exceptionally rare and usually accompanied by MG $(8,9)$. In a review of 377,841 autopsies over a 20 -year period, Wakafuji and Okada (10) found myocarditis in $0.11 \%$ and giant cell myocarditis in $0.007 \%$ of cases. Giant cell myocarditis generally affects young to middle-aged adults of either gender (11). It is characterized by acute heart failure, ventricular arrhythmias or heart block, which progress rapidly to mortality, usually within 10 days (12); although some cases showed a good response to steroid, azathioprine and cyclosporine therapy $(9,13,14)$. However, the mechanisms remain unknown, and it has been speculated in previous reports that antimyocardial or antiskeletal muscle antibodies may be responsible for the abnormalities.

Giant cell myocarditis usually occurs in isolation, but has been reported in association with a variety of autoimmune diseases $(12,15,16)$ and chronic infections $(17,18)$. It is diagnosed in approximately $1 \%$ of patients with thymoma (12). Almost 30 cases have been reported worldwide thus far, and the present patient was the fourth case of thymoma associated with myositis and myocarditis in Japan $(8,19,20)$. However, all four were fatal cases. Tanahashi and colleagues reported that glucocorticoid pulse therapies were not effective (8). Anecdotal reports and animal studies have suggested that immunosuppressive treatment may be effective $(14,21,22)$, but there are no randomized controlled trials to support this theory. There is some evidence to suggest an autoimmune process, and activated T lymphocytes appear to be important for myocarditis development. Myocarditis has been reported to recur in transplanted hearts $(22,23)$. The defective immunity found in patients with thymoma is thought to result in a giant cell immune reaction against myofibers (16). While on chemotherapy for invasive 
thymoma, our patient developed fatal fulminant polymyositis and myocarditis without clinical evidence of MG. An autoimmune mechanism was indicated by the diffuse infiltration of muscles by lymphocytes. Whether chemotherapy triggered this syndrome remains to be determined. Notably, 18 days after receiving the second cycle of chemotherapy, the patient developed high-grade fever. The infection may be correlated with neutropenia caused by chemotherapy, which may trigger the syndrome. Increased awareness of giant cell myocarditis in association with thymoma should lead to early recognition and treatment. This case was the second associated with fatal myocarditis and polymyositis following treatment with carboplatin and paclitaxel (4).

\section{Acknowledgements}

This study was supported in part by the Health and Labour Sciences Research Grant on Intractable Diseases (neuroimmunological diseases) from the Ministry of Health, Labour and Welfare of Japan.

\section{References}

1. Giordano A and Haymondo J: Myasthenia gravis: a report of two cases with necropsy findings. Am J Clin Pathol 14: 253-265, 1944.

2. Burke JS, Medline NM and Katz A: Giant cell myocarditis and myositis. Associated with thymoma and myasthenia gravis. Arch Pathol 88: 359-366, 1969.

3. Namba T, Brunner NG and Grob D: Idiopathic giant cell polymyositis. Report of a case and review of the syndrome. Arch Neurol 31: 27-30, 1974.

4. Gidron A, Quadrini M, Dimov N, et al: Malignant thymoma associated with fatal myocarditis and polymyositis in a 32-year-old woman with a history of hairy cell leukemia. Am J Cin Oncol 29: 213-214, 2006

5. Masaoka A, Monden Y, Nakahara K, et al: Follow-up study of thymomas with special reference to their clinical stages. Cancer 48: 2485-2492, 1981.

6. Kondo K and Monden Y: Therapy for thymic epithelial tumors: a clinical study of 1320 patients from Japan. Ann Thorac Surg 76: $878-885,2003$
7. Venuta F, Rendina EA, Pescarmona EO, et al: Multimodality treatment of thymoma: a prospective study. Ann Thorac Surg 64: 1585-1592, 1997.

8. Tanahashi N, Sato H, Nogawa S, et al: A case report of giant cell myocarditis and myositis observed during the clinical course of invasive thymoma associated with myasthenia gravis. Keio J Med 53: 30-42, 2004.

9. Ko KF, Ho T and Chan KW: Autoimmune chronic active hepatitis and polymyositis in a patient with myasthenia gravis and thymoma. J Neurol Neurosurg Psychiatry 59: 558-559, 1995.

10. Wakafuji S and Okada R: Twenty year autopsy statistics of myocarditis incidence in Japan. Jpn Circ J 50: 1288-1293, 1986.

11. Glennon PE, Petersen ME and Sheppard MN: Fatal giant cell myocarditis after resection of thymoma. Heart 75: 531-532, 1996.

12. Davies MJ, Pomerance A and Teare RD: Idiopathic giant cell myocarditis - a distinctive clinoco-pathological entity. Br Heart J 37: 192-195, 1975.

13. Ren H, Poston RS, Hruban RH, et al: Long survival with giant cell myocarditis. Mod Pathol 6: 402-407, 1993.

14. Desjardins V, Pelletier G, Leung TL and Waters D: Successful treatment of severe heart failure caused by idiopathic giant cell myocarditis. Can J Cardiol 8: 788-792, 1992.

15. Roberts WC, Kehoe JA, Carpenter DF, et al: Cardiac valvular lesions in rheumatoid arthritis. Arch Intern Med 122: 141-146, 1968.

16. Humbert P, Faivre R, Fellman D, et al: Giant cell myocarditis: an autoimmune disease? Am Heart J 115: 485-487, 1988.

17. Reingold IM: Myocardial lesions in disseminated coccidioidomycosis. Am J Clin Pathol 20: 1044-1049, 1950.

18. Buchibinder NA and Robert WC: Left-sided valvular active infective endocarditis: a study of forty-five necropsy patients. Am J Med 53: 20-35, 1972.

19. Suzuki H, Mizuno Y, Kurita T, et al: Rare type myositis and myocarditis revealed in myasthenia gravis with malignant thymoma. Saishin-igaku (in Japanese) 31: 2417-2424, 1976.

20. Tomimoto H, Akiguchi I, Kameyama M, et al: Giant cell myositis and myocarditis associated with myasthenia gravis and thymoma-an autopsy case. Rinsho Shinkeigaku (in Japanese) 25: 688-693, 1985.

21. Zhang S, Kodama M, Hanawa H, et al: Effects of cyclosporine, predonisolone and aspirin on rat autoimmune giant cell myocarditis. J Am Coll Cardiol 21: 1254-1260, 1993.

22. Kong G, Madden B, Spyrou N, et al: Response of recurrent giant cell myocarditis in a transplanted heart to intensive immunosuppression. Eur Heart J 12: 554-557, 1991.

23. Gries W, Farkas D, Winters GL, et al: Giant cell myocarditis: first report of disease recurrence in the transplanted heart. J Heart Lung Transplant 11: 370-374, 1992. 